\title{
Zwei graphentheoretische Probleme
}

\author{
Lisa Sauermann
}

In meiner Dissertation geht es um verschiedene Probleme in extremaler Kombinatorik. Dieser Beitrag diskutiert zwei Resultate, die sich beide um graphentheoretische Fragestellungen drehen.

Die extremale Kombinatorik ist ein sehr lebendiges Gebiet der Mathematik, mit engen Verbindungen zu anderen Gebieten wie der Wahrscheinlichkeitstheorie, der diskreten Geometrie und der analytischen Zahlentheorie, sowie auch zur theoretischen Informatik. Die Ursprünge der extremalen Kombinatorik liegen mehr als hundert Jahre zurück, und das Gebiet hat sich seither fortlaufend weiterentwickelt. Während kombinatorische Probleme traditionell mithilfe rein kombinatorischer Methoden gelöst wurden, nutzte man in der extremalen Kombinatorik im Laufe der Zeit immer mehr Mittel aus anderen Bereichen der Mathematik. Dies begann mit der Verwendung probabilistischer Methoden, insbesondere durch Erdős, doch mittlerweile werden Methoden aus vielen weiteren Gebieten der Mathematik eingesetzt. Meine Doktorarbeit untersucht verschiedene Probleme in extremaler Kombinatorik und verwendet Techniken aus mehreren anderen Gebieten, insbesondere aus der Wahrscheinlichkeitstheorie und der Algebra.

In diesem Beitrag möchte ich zwei Ergebnisse aus meiner Dissertation vorstellen. Das erste beweist eine 25 Jahre alte Vermutung von Erdős, Faudree, Rousseau und Schelp [5] hinsichtlich der Existenz von Teilgraphen mit Minimalgrad $k$ in einem Graphen mit ausreichend vielen Kanten. Einen Spezialfall dieser Vermutung nahm Erdős in eine Liste seiner Lieblingsproblemen in der Graphentheorie [4] auf.

Das zweite in diesem Beitrag diskutierte Ergebnis betrifft die Anzahl der $n$-Knoten-Graphen in bestimmten algebraisch definierten Graphenklassen (wobei die Kanten der Graphen mittels der Vorzeichen einer gegebenen endlichen Liste von Polynomen definiert sind). Viele solcher Graphenklassen treten natürlicherweise in der diskreten Geometrie auf, zum Beispiel die Klasse der Schnittgraphen von Strecken in der Ebene (oder Kreisscheiben in der Ebene). Der Satz von Warren [11] (eine Variante eines Satzes von Milnor [7] und Thom [10]) impliziert eine obere Schranke für die Anzahl der $n$-Knoten-Graphen in einer solchen algebraisch definierten Graphenklasse. Für untere Schranken waren bisher jedoch nur Ad-hoc-Konstruktionen für bestimmte Graphenklassen bekannt. In meiner Dissertation habe ich ein allgemeines Resultat bewiesen, das eine untere Schranke für die Anzahl der $n$-Knoten-Graphen in vielen solchen Graphenklassen zeigt. Diese untere Schranke stimmt im Wesentlichen mit der oberen Schranke überein, die sich aus dem Satz von Warren ergibt. Der Beweis meines Resultats verwendet Werkzeuge aus der algebraischen Geometrie und der Differentialtopologie.
1 Teilgraphen mit Minimalgrad $k$

Probleme der extremalen Kombinatorik beschäftigen sich häufig mit der maximalen oder minimalen Größe kombinatorischer Konfigurationen unter bestimmten Bedingungen. Ein einfaches Beispiel ist die folgende Frage: Was ist die maximale Anzahl von Kanten, die ein $n$-Knoten-Graph haben kann, ohne einen Kreis zu enthalten? Ein Graph ohne einen Kreis wird als Wald bezeichnet, und es ist ein einfacher Fakt (und einer der ersten Sätze in einem einführenden Kurs in Graphentheorie), dass jeder Wald mit $n$ Knoten höchstens $n-1$ Kanten hat. Das bedeutet, dass jeder Graph mit $n$ Knoten und mindestens $n$ Kanten einen Kreis enthalten muss.

In einem Kreis hat jeder Knoten Grad 2. Daher enthält jeder Graph mit $n$ Knoten und mindestens $n$ Kanten einen Teilgraphen, in dem jeder Knoten Grad mindestens 2 hat. Doch was geschieht, wenn wir für eine feste Zahl $k>2$ einen Teilgraphen finden möchten, in dem jeder Knoten Grad mindestens $k$ hat?

Wenn man eine ausreichend große Anzahl von Kanten in einem $n$-Knoten-Graphen hat, ist es auch hier stets der Fall, dass der Graph einen Teilgraphen besitzt, in dem jeder Knoten Grad mindestens $k$ hat (d. h. der Minimalgrad des Teilgraphen ist mindestens $k$ ). Der folgende Fakt, gezeigt 1990 von Erdős, Faudree, Rousseau und Schelp [5], präzisiert dies.

Fakt 1. Jeder Graph mit $n \geq k-1$ Knoten und mindestens $(k-1)(n-k+2)+\left(\begin{array}{c}k-2 \\ 2\end{array}\right)$ Kanten enthält einen Teilgraphen mit Minimalgrad mindestens $k$.

Es ist nicht schwierig, diese Aussage per Induktion nach $n \mathrm{zu}$ beweisen.

Erdős et al. beobachteten auch, dass ihre Schranke in Fakt 1 scharf ist. In der Tat erhält man, wenn man einen vollständigen Graphen mit $k-2$ Knoten und einen Weg mit $n-k+2$ Knoten nimmt und Kanten von jedem Knoten des vollständigen Graphen zu jedem Knoten des Weges hinzufügt, einen Graphen mit $n$ Knoten und $(k-1)(n-k+2)+$ $\left(\begin{array}{c}k-2 \\ 2\end{array}\right)-1$ Kanten, und dieser Graph hat keinen Teilgraphen mit Minimalgrad mindestens $k$ (siehe die linke Seite von Abbildung 1).

Darüber hinaus stellten Erdős et al. auch fest, dass für jedes $n \geq k+1$ ein Graph mit $n$ Knoten und $(k-1)(n-k+2)+$ $\left(\begin{array}{c}k-2 \\ 2\end{array}\right)$ Kanten existiert, der keinen Teilgraphen mit Minimalgrad mindestens $k$ mit weniger als $n$ Knoten hat. Ein solcher Graph ergibt sich, wenn man einen vollständigen Graphen 

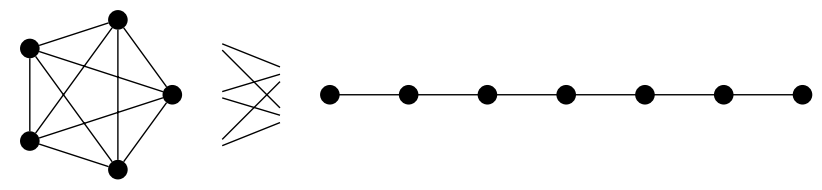

$K_{k-2}$

$P_{n-k+2}$
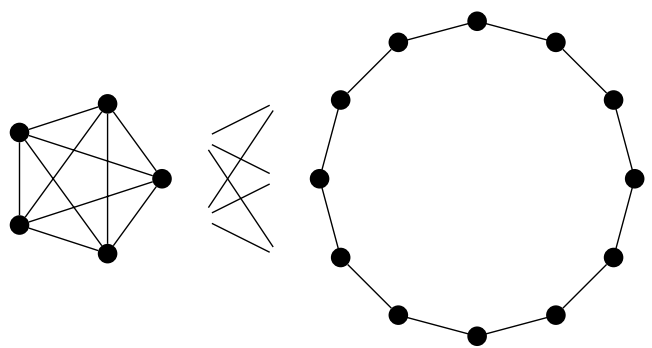

$K_{k-2}$

$C_{n-k+2}$

Abbildung 1. Der Graph auf der linken Seite hat keinen Teilgraphen mit Minimalgrad mindestens $k$. Der Graph auf der rechten Seite besitzt keinen Teilgraphen mit Minimalgrad mindestens $k$ mit weniger als $n$ Knoten.

mit $k-2$ Knoten und einen Kreis mit $n-k+2$ Knoten nimmt und Kanten von jedem Knoten des vollständigen Graphen zu jedem Knoten des Kreises hinzufügt, wie auf der rechten Seite von Abbildung 1 dargestellt.

Was geschieht, wenn wir einen Teilgraphen mit weniger als $n$ Knoten mit Minimalgrad mindestens $k$ finden möchten? Es ist nicht schwer zu zeigen, dass dafür eine zusätzliche Kante, d. h. insgesamt $(k-1)(n-k+2)+\left(\begin{array}{c}k-2 \\ 2\end{array}\right)+1$ Kanten, ausreichend ist.

Erdős et al. vermuteten aber, dass eine zusätzliche Kante nicht nur die Existenz eines Teilgraphen mit Minimalgrad mindestens $k$ mit weniger als $n$ Knoten garantiert, sondern sogar die Existenz eines solchen Teilgraphen mit deutlich weniger als $n$ Knoten:

Vermutung 2. Für jedes $k \geq 2$ existiert $\varepsilon_{k}>0$, sodass jeder Graph mit $n \geq k-1$ Knoten und mindestens $(k-1)(n-k+$ 2) $+\left(\begin{array}{c}k-2 \\ 2\end{array}\right)+1$ Kanten einen Teilgraphen mit höchstens $\left(1-\varepsilon_{k}\right) n$ Knoten und mit Minimalgrad mindestens $k$ enthält.

Erdős nahm diese Vermutung im Spezialfall $k=3$ in eine Liste seiner Lieblingsprobleme in der Graphentheorie auf [4, S. 13]. Fortschritte in Richtung der Vermutung 2 machten sowohl Erdős et al. in ihrem ursprünglichen Artikel [5], in dem sie die Existenz eines solchen Teilgraphen mit höchstens $n-\varepsilon_{k} \sqrt{n}$ Knoten bewiesen, als auch Mousset, Noever und Škorić [8], die die Schranke zu $n-\varepsilon_{k} n / \log n$ verbesserten.

In meiner Dissertation habe ich Vermutung 2 bewiesen. Ich habe dabei die Ideen von Erdős et al. und von Mousset et al. genutzt und erweitert. Die Details des Beweises sind leider ziemlich technisch. Die Grundidee besteht darin, einigen der Knoten des Graphen in solcher Weise Farben zuzuordnen, dass man beim Entfernen aller Knoten einer Farbe stets einen Teilgraphen mit Minimalgrad mindestens $k$ erhält. Wenn man dabei ausreichend viele Knoten färbt (aber die Anzahl der Farben nur von $k$ abhängt), dann muss eine der Farbklassen aus mindestens $\varepsilon_{k} n$ Knoten bestehen, und man erhält beim Entfernen dieser Farbklasse einen Teilgraphen mit Minimalgrad mindestens $k$ mit höchstens $\left(1-\varepsilon_{k}\right) n$ Knoten.
Eine solche Färbung wird in einem iterativen Prozess konstruiert, wobei bei jedem Iterationsschritt die Techniken von Mousset, Noever und Škorić [8] angewendet werden. Doch um den iterativen Prozess weiterführen zu können, muss man eine viel bessere Kontrolle darüber haben, was genau bei jedem Schritt geschieht.

Mein Beweis zeigt, dass Vermutung 2 mit $\varepsilon_{k}=10^{-4} k^{-3}$ stimmt. Andererseits kann man Beispiele finden, die für den größtmöglichen Wert von $\varepsilon_{k}$ in Vermutung 2 eine obere Schranke von $\varepsilon_{k} \leq O\left(k^{-2}\right)$ zeigen. Meiner Meinung nach ist es ein interessantes Problem, das Wachstumsverhalten des größtmöglichen Wertes für $\varepsilon_{k}$ in Vermutung 2 zu bestimmen:

Frage 3. Was ist der größte Wert für $\varepsilon_{k}$, für den Vermutung 2 zutrifft?

Zählen algebraisch definierter Graphen

Nehmen wir an, wir haben $n$ Strecken in der Ebene, die von 1 bis $n$ nummeriert sind. Dann können wir ihren Schnittgraphen als den Graphen mit Knotenmenge $\{1, \ldots, n\}$ definieren, in dem zwei Knoten genau dann durch eine Kante verbunden sind, wenn sich die entsprechenden Strecken schneiden. Abbildung 2 veranschaulicht dies.

Auf die gleiche Weise können wir Schnittgraphen anderer geometrischer Objekte definieren, zum Beispiel von Kreisscheiben in der Ebene oder von $d$-dimensionalen $\mathrm{Ku}$ geln in $\mathbb{R}^{d}$. Schnittgraphen wurden intensiv untersucht und sind in verschiedenen praktischen Anwendungen von Bedeutung (z. B. im Database Mining [2], bei der Modellierung von Rundfunknetzwerken [3] und sogar in der Genetik $[1,9])$.

Es gibt noch viele weitere Graphenklassen, die aus der diskreten Geometrie abgeleitet werden können. Zum Beispiel können wir für $n$ disjunkte (starre) Kreise in $\mathbb{R}^{3}$, die von 1 bis $n$ nummeriert sind, ihren Verschlingungsgraphen als den Graphen auf der Knotenmenge $\{1, \ldots, n\}$ definieren, 
in dem zwei Knoten genau dann durch eine Kante verbunden sind, wenn die dazugehörigen Kreise in $\mathbb{R}^{3}$ ineinander verschlungen sind.

Eine sehr naheliegende Frage hinsichtlich dieser Graphenklassen lautet: Wie viele Graphen (mit der Knotenmenge $\{1, \ldots, n\})$ enthält jede dieser Graphenklassen? Mit anderen Worten: Wie viele Graphen mit der Knotenmenge $\{1, \ldots, n\}$ können sich als Schnittgraphen von $n$ nummerierten Strecken in der Ebene ergeben? Und wie viele können sich als Schnittgraphen von $n$ nummerierten offenen Kreisscheiben in der Ebene ergeben? Oder als Verschlingungsgraphen von $n$ nummerierten disjunkten Kreisen in $\mathbb{R}^{3}$ ?

Für diese Fragen ist eine algebraische Perspektive hilfreich. Für jede dieser Graphenklassen können wir die entsprechenden geometrischen Objekte (z. B. die Strecken oder Kreisscheiben in der Ebene) durch Punkte in $\mathbb{R}^{d}$ für geeignetes $d$ parametrisieren. Die jeweiligen geometrischen Beziehungen (z. B. Überschneidungen oder Verschlingungen) können wir dann durch polynomielle Bedingungen charakterisieren. Zum Beispiel entspricht eine offene Kreisscheibe in der Ebene einem Punkt $(x, y, r) \in \mathbb{R}^{3}$ mit $r>0$ (wobei $(x, y)$ der Mittelpunkt und $r>0$ der Radius ist), und zwei den Punkten $(x, y, r)$ und $\left(x^{\prime}, y^{\prime}, r^{\prime}\right)$ entsprechende offene Kreisscheiben überschneiden sich genau dann, wenn $\left(x-x^{\prime}\right)^{2}+\left(y-y^{\prime}\right)^{2}-\left(r+r^{\prime}\right)^{2}<0$. Für andere Graphenklassen (z. B. für Schnittgraphen von Strecken in der Ebene) benötigt man mehr als ein Polynom, um die gewünschten geometrischen Beziehungen zu charakterisieren. Aber für viele Klassen von Graphen, die in der diskreten Geometrie auftreten, können die gewünschten geometrischen Beziehungen mittels der Vorzeichen einer endlichen Liste von Polynomen algebraisch charakterisiert werden.

Mit dieser algebraischen Perspektive impliziert der Satz von Warren [11] (eine Variante eines Satzes von Milnor [7] und Thom [10]) eine obere Schranke für die Anzahl der Graphen mit Knotenmenge $\{1, \ldots, n\}$ in jeder der oben betrachteten Graphenklassen. Allgemeiner gesagt erhält man eine solche obere Schranke für jede Klasse von Graphen, bei der die Kanten algebraisch mittels der Vorzeichen einer gegebenen endlichen Liste von Polynomen definiert sind.
In meiner Dissertation habe ich eine untere Schranke für die Anzahl der Graphen mit Knotenmenge $\{1, \ldots, n\}$ in einer solchen Graphenklasse bewiesen (unter der Annahme bestimmter Bedingungen für die gegebene endliche Liste von Polynomen, die zur Definition der Kanten verwendetet wird). Diese untere Schranke entspricht im Wesentlichen der oberen Schranke, die sich aus dem Satz von Warren ergibt, ist also im Grunde scharf.

Die folgenden Aussagen, die die oben gestellten Fragen beantworten, ergeben sich als einfache Anwendungen dieses Resultats: Die Anzahl der Graphen mit Knotenmenge $\{1, \ldots, n\}$, die als Schnittgraphen von $n$ nummerierten Strecken in der Ebene dargestellt werden können, ist $n^{(4+o(1)) n}$. Die Anzahl der Schnittgraphen von $n$ nummerierten offenen Kreisscheiben in der Ebene ist $n^{(3+o(1)) n}$. Und die Anzahl der Verschlingungsgraphen von $n$ nummerierten disjunkten (starren) Kreisen in $\mathbb{R}^{3}$ ist $n^{(6+o(1)) n}$. Es ergeben sich auch zahlreiche weitere Anwendungen für die Anzahl der Graphen mit Knotenmenge $\{1, \ldots, n\}$ in vielen anderen Graphenklassen, die in der diskreten Geometrie auftreten.

Die obere Schranke (die sich aus dem Satz von Warren ergibt) war bereits seit mehr als dreißig Jahren bekannt. Für einige dieser Anwendungen war auch die untere Schranke bekannt, aber alle bekannten unteren Schranken wurden durch (manchmal recht komplizierte) Ad-hocKonstruktionen für spezifische Familien von geometrischen Objekten gezeigt. Zum Beispiel bewies Fox durch eine spezifische Konstruktion für Strecken in der Ebene, dass die Anzahl der Schnittgraphen von $n$ nummerierten Strecken in der Ebene mindestens $n^{(4+o(1)) n}$ beträgt. McDiarmid und Müller [6] zeigten mittels einer spezifischen Konstruktion für Kreisscheiben in der Ebene, dass die Anzahl der Schnittgraphen von $n$ nummerierten offenen Kreisscheiben in der Ebene mindestens $n^{(3+o(1)) n}$ beträgt. Keine dieser Konstruktionen lässt sich ohne Weiteres auf andere Graphenklassen verallgemeinern. Im Gegensatz dazu ist das Ergebnis in meiner Dissertation deutlich allgemeiner, und impliziert insbesondere diese zuvor bekannten unteren Schranken für spezifische Fälle als einfache Anwendungen (und impliziert außerdem neue untere Schranken für viele andere Fälle,
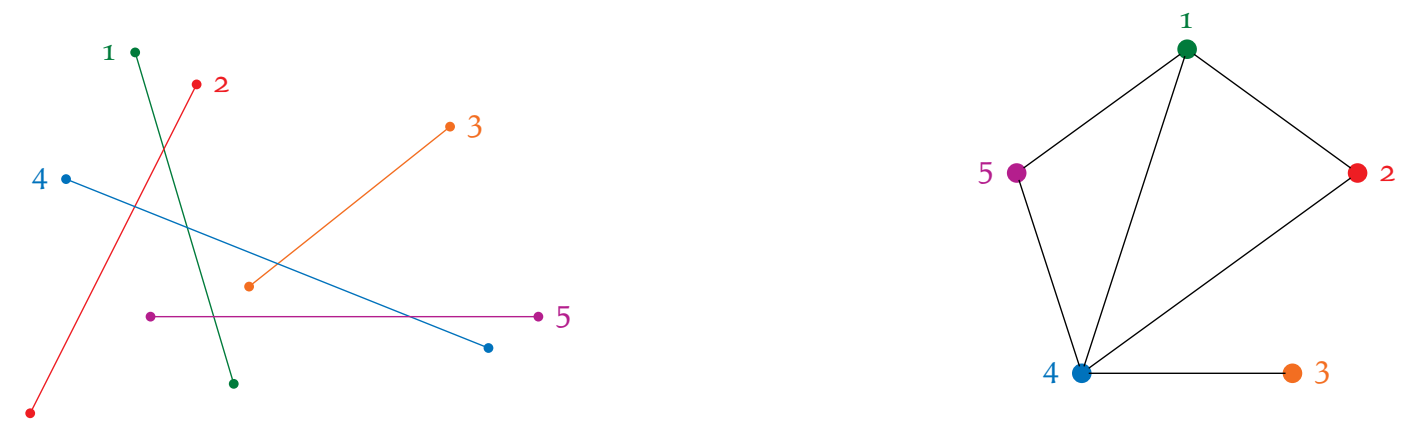

Abbildung 2. Die linke Seite zeigt eine Konstellation von fünf Strecken, die von 1 bis 5 nummeriert sind. Der entsprechende Schnittgraph ist auf der rechten Seite dargestellt. 
zum Beispiel für viele andere Klassen von Schnittgraphen und auch für verschiedene Familien von in der diskreten Geometrie auftretenden Teilmengenordnungen).

Die exakte Formulierung dieses Resultats in meiner Dissertation ist leider etwas technisch (und wahrscheinlich nicht sehr aufschlussreich), sodass darauf hier verzichtet wird. Zusätzlich zu kombinatorischen Argumenten verwendet der Beweis des Resultats Mittel aus der algebraischen Geometrie und der Differentialtopologie.

\section{References}

[1] S. Benzer, On the topology of the genetic fine structure. Proc. Natl. Acad. Sci. USA 45 (1959), 1607-1620.

[2] P. Berman, B. DasGupta, S. Muthukrishnan, and S. Ramaswami, Efficient approximation algorithms for tiling and packing problems with rectangles. J. Algorithms 41 (2001), 443-470.

[3] B. N. Clark, C. J. Colbourn, and D. S. Johnson, Unit disk graphs. Discrete Math. 86 (1990), 165-177.
[4] P. Erdős, Some of my favorite solved and unsolved problems in graph theory. Quaestiones Math. 16 (1993), 333-350.

[5] P. Erdős, R. J. Faudree, C. C. Rousseau, and R. H. Schelp, Subgraphs of minimal degree $k$. Discrete Math. 85 (1990), 53-58.

[6] C. McDiarmid and T. Müller, The number of disk graphs. European J. Combin. 35 (2014), 413-431.

[7] J. Milnor, On the Betti numbers of real varieties. Proc. Amer. Math. Soc. 15 (1964), 275-280.

[8] F. Mousset, A. Noever, and N. Škorić, Smaller subgraphs of minimum degree k. Electron. J. Combin. 24 (2017), Paper 4.9, 8 pp.

[9] J.P. Spinrad, Efficient Graph Representations. Fields Institute Monographs, 19, American Mathematical Society, Providence, RI, 2003.

[10] R. Thom, Sur l'homologie des variétés algébriques réelles. In Differential and Combinatorial Topology, pages $255^{-265}$, Princeton University Press, 1965.

[11] H.E. Warren, Lower bounds for approximation by nonlinear manifolds. Trans. Amer. Math. Soc. 133 (1968), 167-178.
Dr. Lisa Sauermann, Stanford University,
Department of Mathematics, Building 380,
Stanford, California 94305, USA
lsauerma@stanford.edu

Lisa Sauermann ist derzeit Szegö Assitant Professor an der Stanford University in Kalifornien. Ihr Forschungsschwerpunkt liegt in extremaler Kombinatorik. Sie hat ihren Bachelorabschluss im Jahr 2014 an der Universität Bonn abgelegt und 2019 an der Stanford University promoviert. 